La mutation des emplois hautement qualifiés dans l'agglomération transfrontalière strasbourgeoise ( The mutation of highly qualified labour in the border agglomeration of Strasbourg) Jörg Wendel

Citer ce document / Cite this document :

Wendel Jörg. La mutation des emplois hautement qualifiés dans l'agglomération transfrontalière strasbourgeoise ( The mutation of highly qualified labour in the border agglomeration of Strasbourg). In: Bulletin de l'Association de géographes français, 81e année, 2004-4 ( décembre). Les frontières dans la ville / Problématique urbaine en Suisse. pp. 596-620; doi : https://doi.org/10.3406/bagf.2004.2425

https://www.persee.fr/doc/bagf_0004-5322_2004_num_81_4_2425

Fichier pdf généré le 25/04/2018 


\begin{abstract}
In the last decade the agglomeration of Strasbourg has continually expanded and confirmed its importance as a salient pole in European science and technology. Parallel to the economic spread in certain districts and surroundings of Strasbourg, the economic and social situation has dramatically aggravated in other parts of the agglomeration. Similar to the large cities in the United States, there are also in Strasbourg parallel societies which develop completely disconnected from each other. At the edge of the urban perimeter, surrounded by good situated quarters of the city centre and the wealthy suburban communities, a gradually closing belt of underprivileged districts is emerging. Despite of their immediate proximity to areas of growth, these districts are in reality largely disconnected to the overall very positive economic and social development. They risk falling into a complete functional and economic insignificance.
\end{abstract}

\title{
Résumé
}

Depuis une décennie environ, I' agglomération de Strasbourg a fortement affirmé et consolidé sa position européenne en tant que pôle scientifique et technologique européen. Toutefois, parallèlement au déploiement économique de certains de ses quartiers et communes aux environs de Strasbourg, la situation économique et sociale s'est détériorée de façon dramatique dans d'autres parties de l'agglomération. A la manière de l'évolution observée dans les grandes villes des Etats-Unis, se développent aussi visiblement à Strasbourg, côte à côte, mais contradictoirement, des sociétés déconnectées les unes des autres. Au bord du périmètre urbain, inséré entre les quartiers relativement cossus du centre-ville et les communes suburbaines prospères, est en train de se structurer une ceinture de quartiers en retard de développement. En dépit de leur proximité immédiate par rapport aux pôles de croissance, ces quartiers sont en effet largement déconnectés de l'évolution socioéconomique, dans I 'ensemble très positive. Ils courent le risque de sombrer dans une insignifiance fonctionnelle et économique quasi-complète. 


\title{
La mutation des emplois hautement qualifiés dans l'agglomération transfrontalière strasbourgeoise
}

\author{
(THE MUTATION OF HIGHLY QUALIFIED LABOUR \\ IN THE BORDER AGGLOMERATION OF STRASBOURG)
}

\section{Jörg WENDEL*}

RÉSUMÉ. - Depuis une décennie environ, l'agglomération de Strasbourg a fortement affirmé et consolidé sa position européenne en tant que pôle scientifique et technologique européen. Toutefois, parallèlement au déploiement économique de certains de ses quartiers et communes aux environs de Strasbourg, la situation économique et sociale s'est détériorée de façon dramatique dans d'autres parties de l'agglomération. A la manière de l'évolution observée dans les grandes villes des Etats-Unis, se développent aussi visiblement à Strasbourg, côte à côte, mais contradictoirement, des sociétés déconnectées les unes des autres. Au bord du périmètre urbain, inséré entre les quartiers relativement cossus du centre-ville et les communes suburbaines prospères, est en train de se structurer une ceinture de quartiers en retard de développement. En dépit de leur proximité immédiate par rapport aux pôles de croissance, ces quartiers sont en effet largement déconnectés de l'évolution socio-économique, dans l'ensemble très positive. Ils courent le risque de sombrer dans une insignifiance fonctionnelle et économique quasi-complète.

Mots-clés: hautes technologies, développement scientifique, fractures socio-économiques spatiales, agglomération de Strasbourg.

ABSTRACT. - In the last decade the agglomeration of Strasbourg has continually expanded and confirmed its importance as a salient pole in European science and technology. Parallel to the economic spread in certain districts and surroundings of Strasbourg, the economic and social situation has dramatically aggravated in

\footnotetext{
* Assistant-Doctorant à l'Institut de Géographie de l'Université de Bâle.
} 
other parts of the agglomeration. Similar to the large cities in the United States, there are also in Strasbourg parallel societies which develop completely disconnected from each other. At the edge of the urban perimeter, surrounded by good situated quarters of the city centre and the wealthy suburban communities, a gradually closing belt of underprivileged districts is emerging. Despite of their immediate proximity to areas of growth, these districts are in reality largely disconnected to the overall very positive economic and social development. They risk falling into a complete functional and economic insignificance.

Key words: high technologies, scientific development, socio-economic spatial fractures, agglomeration of Strasbourg.

Au cours des dernières années, un certain nombre de publications portant sur la géographie économique ont fait état de la nécessité de prendre en compte l'aptitude entrepreneuriale en matière d'innovation, ainsi que la formation de réseaux, en tant que moteurs du développement économique régional, à la fois en ce qui concerne l'implantation et la promotion de certaines branches d'activités (Butzin, 2000; Koschatzy et al., 2001 ; Strambach, 2002). Les milieux politiques et ceux chargés de l'aménagement urbain et régional souhaitent notamment que ces nouvelles tendances conduisent simultanément à une amélioration de la situation économique et à une restructuration sociale adéquate à l'intérieur de la région urbaine.

\section{Régions urbaines innovantes et secteurs négligés en centre-ville}

Les expériences réalisées dans les régions pionnières dans le domaine de l'innovation économique - tels la Silicon Valley, le Corridor londonien M4 ou la région de Stuttgart - montrent que la concentration spatiale d'entreprises orientées vers la recherche-développement, d'établissements de formation, de firmes à base de hautes technologies en information et en communication, favorise de façon décisive l'implantation et la création d'entreprises innovantes (par exemple en fonction du «spinn-off» des universités ou des exigences très pointues en services spéciaux). Les mécanismes et effets de cette mise en réseau de proximité spatiale ne sont cependant pas mesurables ni statistiquement ni empiriquement; aucune appréciation d'ensemble et définitive n'est possible jusqu'à présent (Kulke, 1998, p. 125 ss ; Aydalot, 1986).

En attendant, il est pourtant difficile de nier les répercussions positives des hautes technologies et des services de haut niveau sur le marché local et régional (Floeting, 1986), ainsi que les conséquences négatives qui résultent de cette mutation pour les salariés peu qualifiés (Keim, 1999, p. 12 ss.). Nous 
constatons que la suppression d'emplois dans les secteurs de production de marchandises comme dans les services «vieillis» (assurances, «call centers»...) n'est de loin pas compensée par les emplois nouveaux créés à la faveur de l'émergence de postes de travail hautement qualifiés et hautement spécialisés dans les secteurs de production, de services et de recherche d'avant-garde. Face à ce contraste, Rifkin parle de «la fin du travail» (Rifkin, 1995). Selon la Commission de Bavière et de Saxe chargée des questions d'avenir, le travail conçu comme une source de revenu garanti pour subvenir aux besoins existentiels et à la sécurité sociale est révolu au profit de nouveaux modèles de temps de travail et de formes d'activités (Kommission für Zukunftsfragen, 1996). Cette évolution est appelée à pénaliser de plus en plus les salariés peu qualifiés. Les salariés hautement qualifiés par contre parviendront dans l'ensemble à s'affirmer professionnellement dans les nouveaux domaines d'activité et à assurer leur existence, quoique au prix d'imposants efforts de promotion (Knuth et.al., 2001, p. 3).

Nous observons donc, parallèlement à la consolidation économique d'une partie de la population concernée par la montée des nouvelles activités, un affaiblissement économique et social de l'autre partie de la population. Cette constatation a inévitablement comme conséquence l'hypothèse d'une relation de causalité entre la dégradation socio-économique de certains secteurs urbains et la concentration d'emplois hautement qualifiés sur d'autres aires de la région urbaine.

L'évolution vers des emplois hautement qualifiés fait apparaître une constante spatiale fondée sur des modèles résidentiels spécifiques inhérents aux nouveaux centres de management, d'affaires et de technologies avancées. Tandis que les centres de management et d'affaires se concentrent de préférence dans les centres-villes traditionnels ou dans certains de leurs quartiers (une partie aussi des établissements de recherche-développement, qui se localisent près des emplacements universitaires traditionnels), les équipements nouveaux liés aux hautes technologies -tels que les parcs technologiques et les centres de recherche-, les firmes de haut niveau de services nécessitant des superficies étendues, de même que les unités de technologies avancées en général, s'implantent de préférence à la périphérie de l'agglomération. Entre les quartiers extérieurs prospères et les quartiers revalorisés du centre-ville se localise un espace négligé et désavantagé (Schmitz, 2001, p. 138). Dans cette optique, Krätke parle d'une ville résiduelle («Reststadt»), menacée par une insignifiance économique et la dégradation sociale (Krätke, 1991).

Les processus de ségrégation métropolitaine apparus récemment ont été décrits amplement et de façon détaillée dans les publications de géographie humaine et de sociologie, surtout à partir des grandes villes américaines, mais 
aussi à l'appui d'exemples se rapportant aux régions métropolitaines européennes (Keim, 1999; O'Loughlin \& Friedrichs, 1996); voir aussi Sandner, dans le présent cahier). Le cas des USA montre nettement que l'évolution s'oriente vers une ville bipolaire, une «ville sans classe moyenne» (Schmitz, 2001, p. 139). Dans les métropoles européennes, cette tendance n'est toutefois observable que dans une mesure très faible.

Seule la sociologie ou presque, s'est saisie jusqu'à présent de cette problématique, à savoir la segmentation du marché du travail en marchés partiels autonomes, dans le cadre de la différenciation économique croissante en matière socio-spatiale et de la division du travail. Cette approche est complétée par la recherche sociologique préoccupćc par les processus de verrouillage social inhérents notamment à la ségrégation féminine induite par le marché du travail (Graß1, 2000, p. 37/38). Ces phénomènes ne trouvent pas encore un intérêt majeur en géographie.

Pour contribuer à parer cette lacune, notre étude vise à appuyer notre démonstration sur l'exemple de l'agglomération strasbourgeoise, transfrontalière et binationale, à vocation internationale. Nous avons recours à certaines branches-clés («key branches») de l'activité économique, aux caractéristiques socio-économiques et socio-démographiques appropriées.

Après une courte présentation de l'espace analysé, nous ferons ressortir l'évolution et la répartition spatiale de sites attribués aux établissements de haute technologie, ainsi que l'offre d'emplois propre aux branches-clés concernées. Nous comparerons ensuite les modèles de répartition entre eux.

Les bases statistiques sont malheureusement insuffisantes pour donner un aperçu exhaustif des relations causales analysées. Notre démarche ne peut être considérée que comme une incitation à se préoccuper de la problématique soulevée, factuellement et méthodologiquement.

\section{L'Eurométropole de Strasbourg : une étude de cas expressive}

La mondialisation croissante de l'économie et le renforcement consécutif de la compétition métropolitaine en Europe, tant à l'intérieur de l'Union Européenne qu'à l'extérieur, ont accentué la réflexion relative aux eurométropoles. Dans leur cheminement concurrentiel, les villes et régions urbaines découvrent de manière grandissante leurs atouts les plus typiques en vue d'un positionnement aussi favorable que possible parmi les sites particulièrement attractifs (Blotevogel, 2001).

Le réseau métropolitain européen existant, qui bénéficie de l'aval de la Commission de l'Union européenne, vise à conforter les positions acquises. 
La mise en réseau des régions par des axes interrégionaux et internationaux de transport appropriés, tels que les réseaux de trains à grande vitesse, les liaisons aéroportuaires et un réseau routier performant, vise à la fois la promotion de l'interaction économique entre les régions, la mobilité individuelle, les échanges culturels et l'intégration européenne (Behrend \& Kruse, 2001).

La région métropolitaine de Strasbourg offre à cet égard une exceptionnelle illustration. Occupant une position relativement centrale dans la région alsacienne fortement urbanisée, l'ouverture frontalière lui confère une certaine centralité européenne dans l'espace du Rhin supérieur méridional à vocation trinationale (Wackermann, 2000). Elle constitue en outre la seule métropole transfrontalière de l'Union européenne.

Dans le contexte national, Strasbourg compte parmi les sept métropoles qui dominent le réseau urbain français et se situe directement derrière Toulouse, Lyon et Marseille (Julien, 1996). Dans le cadre de l'aménagement du territoire français, Strasbourg n'est plus considérée comme une ville périphérique nationale, mais comme une centralité européenne économique et politique, qui dispose d'une réelle force économique et d'une aptitude élevée à l'innovation (Nonn, 1999). Cette position est renforcée par la création d'un district transfrontalier Strasbourg-Kehl, par suite d'une décision intergouvernementale prise lors de la célébration, à Paris, en 2003, du cinquantenaire des traités de l'Elysée. La recherche en cours, sur la compétitivité des métropoles européennes, induite par la Commission de Bruxelles, et confiée jusqu'à son terme (2004) à Gabriel Wackermann et à moi-même, en ce qui concerne le cas transfrontalier strasbourgeois, permet de scruter de façon relativement fine la problématique soulevée.

La partie négative de cette ville européenne, ses handicaps sociaux concentrés dans certains quartiers de l'agglomération, tels que ceux de Hautepierre, de Cronenbourg, du Neuhof, est également connue nationalement et internationalement par les flashes médiatiques, mettant l'accent sur les voitures incendiées, la population en provenance de l'immigration, une certaine décomposition socio-spatiale (cf. la contribution de Sahli \& Schneider - dans le même numéro).

\section{Evolution générale et mise en réseau croissante de l'espace métropolitain}

Au cours de la dernière décennie, la seule ville de Strasbourg a connu une croissance substantielle. Le nombre d'habitants et d'actifs y a augmenté sensiblement (tableau 1). 
Tableau 1. Population/densité démographique et offre/densité d'emplois à Strasbourg et dans la Communauté urbaine de Strasbourg (CUS).

\begin{tabular}{|c|c|c|c|c|}
\hline & Année & Strasbourg & CUS & $\begin{array}{c}\text { Part de Strasbourg } \\
\text { à la CUS) }\end{array}$ \\
\hline \multirow{2}{*}{$\begin{array}{l}\text { Nombre } \\
\text { d'hab. }\end{array}$} & 1990 & 252338 & 423712 & $59,5 \%$ \\
\hline & 1999 & $264115(+4,6 \%)$ & $451240(+6,4 \%)$ & $58,5 \%$ \\
\hline \multirow{2}{*}{$\begin{array}{l}\text { Densité } \\
\text { démogra- } \\
\text { phique } \\
\text { (hab./ } / \mathrm{km}^{2} \text { ) }\end{array}$} & 1990 & 3224 & 1385 & \\
\hline & 1999 & $3374(+4,6 \%)$ & $1475(+6,5 \%)$ & \\
\hline \multirow{2}{*}{$\begin{array}{l}\text { Nombre } \\
\text { d'actifs }\end{array}$} & 1990 & 100807 & 179407 & $56,2 \%$ \\
\hline & 1999 & $102649(+1,8 \%)$ & $188587(+5,1 \%)$ & $54,4 \%$ \\
\hline $\begin{array}{l}\text { Nombre } \\
\text { emplois }\end{array}$ & 1999 & 151437 & 224449 & $67,5 \%$ \\
\hline \multirow{2}{*}{$\begin{array}{l}\text { Densité } \\
\text { d'actifs }\end{array}$} & 1990 & 1288 & 586 & \\
\hline & 1999 & $1311(+1,8 \%)$ & $616(+5,1 \%)$ & \\
\hline
\end{tabular}

Source: INSEE.

Cette évolution est en contradiction directe avec celle observée dans les autres métropoles de notre panel Comet (Amsterdam, Barcelone, Berlin, Bruxelles, Copenhague, Vienne), notamment avec Berlin (Scherf, 1998). Si l'on y inclut les 26 autres communes de la Communauté urbaine de Strasbourg, la croissance économique et le potentiel qui est étroitement lié à celle-ci apparaissent de façon encore plus marquante. Strasbourg et son espace alentour ont ainsi enregistré à la fois une progression démographique et une accentuation de l'offre d'emplois. L'augmentation et l'élargissement de l'offre d'emplois est cependant très variée d'une commune à l'autre. Ils sont plus importants dans les communes localisées autour de Strasbourg qu'à Strasbourg même. De 1990 à 1999, Geispolsheim et Illkirch-Graffenstaden ont ainsi bénéficié d'une création d'emplois supérieure à celle de Strasbourg. La position du noyau urbain est toutefois demeurée stable, avec plus de la moitié de la population et des emplois de la CUS. Strasbourg ne perd pas encore en importance face au restant de la CUS.

Nous allons aborder maintenant l'analyse de l'évolution des emplois dans certains secteurs économiques, en particulier celle qui est propre à des branches-clés expressives de la mutation en cours. 
Cette évolution s'inscrit dans la mise en réseau précoce de Strasbourg avec son environnement spatial, un environnement qui correspond essentiellement au territoire de l'actuelle CUS. La figure 1 montre le développement de la «tache urbaine»en 1957, 1984 et 1990. En ce qui concerne 1999, j'ai extrait la totalité de la surface bâtie, c'est-à-dire non seulement les aires consacrées aux lotissements, mais aussi aux voies de communication et aux activités industrielles, de l'image satellite que j'ai réalisée pour l'ensemble de l'agglomération transfrontalière de Strasbourg-Ortenau. Cette représentation cartographique fait ressortir nettement l'existence de corridors typiques de croissance le long des axes majeurs de communication et en direction des sites industriels et artisanaux, tel celui localisé autour de l'aéroport de Strasbourg-Entzheim. Du côté allemand, nous ne disposons malheureusement pas encore des statistiques relatives à 1999, nécessaires à une comparaison transfrontalière. Il n'est donc pas encore possible de donner des précisions sur la jonction physique de l'agglomération binationale. La figure 1 révèle néanmoins que l'agglomération de Strasbourg s'est développée des deux côtés du Rhin, quoique essentiellement sur la rive gauche française.

Parallèlement aux liens morphologiques de Strasbourg avec son espace alentour, nous pouvons observer une intense articulation réciproque des flux migratoires intracommunautaire, un réseau qui ressort nettement du Schéma de cohérence territoriale de la région de Strasbourg (SCOTERS), et qui est illustré par le tableau 2, ainsi que par la figure 2 .

En 1999, Strasbourg a attiré environ 53000 migrants pendulaires professionnels domiciliés dans le périmètre du SCOT. Ce nombre, en voie d'accroissement, est nourri à environ $73 \%$ par la CUS, c'est-à-dire par un rayon maximal de $15 \mathrm{~km}$ de distance. Ce taux élevé témoigne d'une connexion étroite et intense entre le noyau urbain de Strasbourg et son environnement spatial immédiat. Il est significatif que plus d'un tiers des immigrants pendulaires sont domiciliés dans les grandes communes alentour de Schiltigheim $(15 \%)$, Illkirch-Graffenstaden (10\%), Lingolsheim (9\%) et Bischheim $(8 \%)$.

Les flux centrifuges de Strasbourg sont encore plus concentrés que les flux centripètes. Plus de $40 \%$ des migrants vers l'extérieur de Strasbourg ont leur lieu de travail à Illkirch-Graffenstaden ou Schiltigheim. Il est à supposer qu'il s'agit là aussi pour une part importante de salariés hautement qualifiés travaillant dans les établissements de recherche et du tertiaire supérieur en général.

Si le nombre de migrants en provenance d'Allemagne est insignifiant dans ce contexte, 6000 à 7000 salariés domiciliés en France vont par contre 


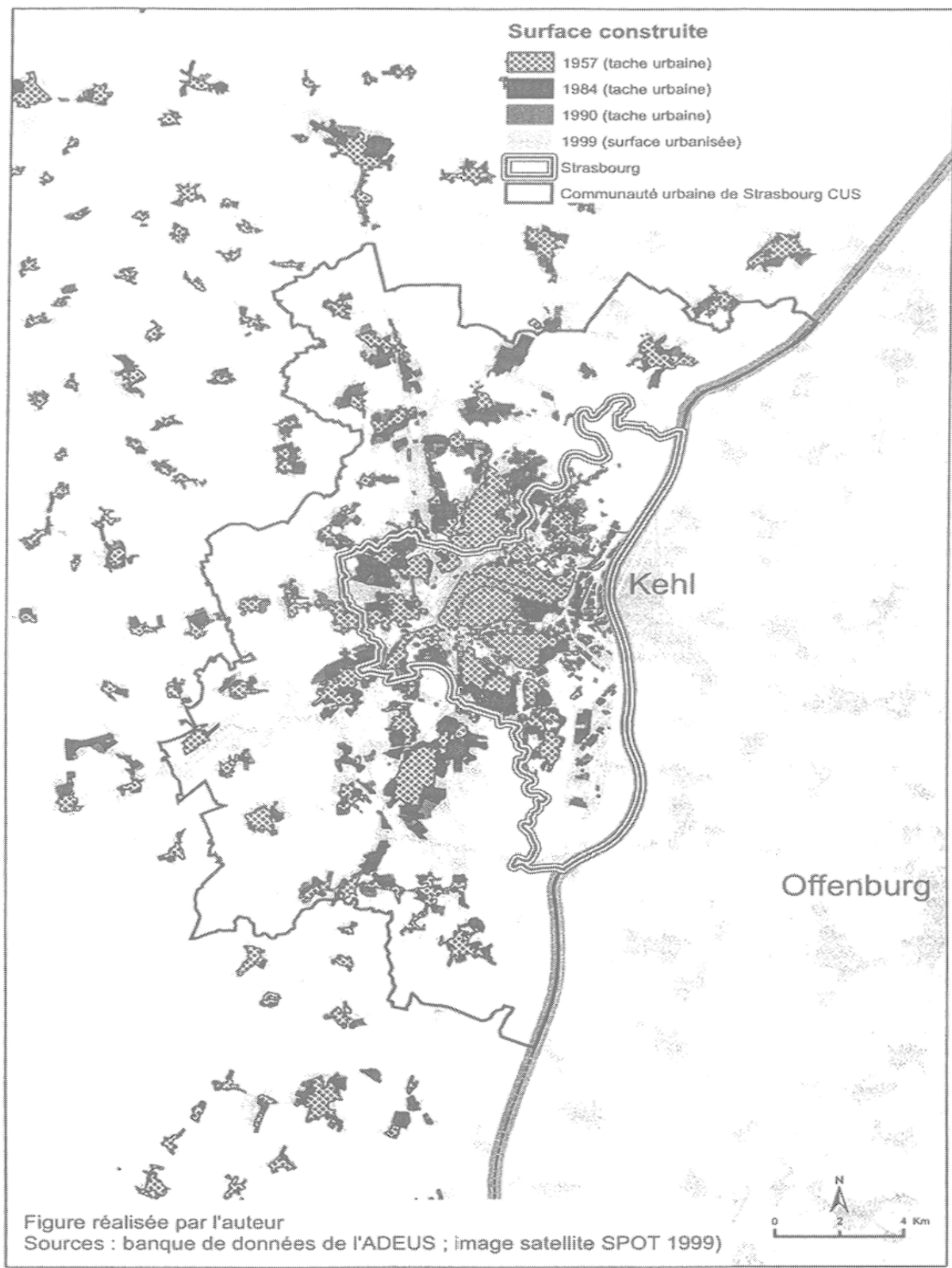

Fig. 1. Evolution de l'espace de peuplement dans l'agglomération de Strasbourg. 
Tableau 2. Flux migratoires pendulaires entre Strasbourg et le restant de la CUS/SCOTERS (1999).

\begin{tabular}{|l|c|c|c|c|}
\hline & Immigrants & Emigrants & Solde migratoire & Pourcentage \\
\hline & pendulaires & pendulaires & & \\
\hline CUS & 38556 & 14112 & +24444 & $79,9 \%$ \\
\hline SCOTERS & 52915 & 16707 & +36208 & $27,1 \%$ \\
\hline
\end{tabular}

Source: INSEE.

travailler quotidiennement en Allemagne, attirés par des salaires plus élevés offerts par le Bade-Wurtemberg dans le secteur économique secondaire.

Si le Projet européen de recherche COMET s'est contenté de délimiter morphologiquement l'agglomération selon la méthode NUREC (fig. 3), ne permettant pas de constater s'il existait des articulations fonctionnelles entre le noyau urbain et son espace alentour, il est cependant très important de connaître la dynamique induite par les réseaux migratoires, surtout à l'époque d'une société fondée de plus en plus sur les technologies de l'information et de la communication. L'approche selon la méthode européenne NUREC ne constitue ainsi qu'une première étape (Wackermann, 2001). La problématique approfondie d'ensemble donne lieu, à l'heure actuelle, à des investigations conduites à l'Institut de géographie de Bâle. Nous livrons ci-après les résultats-clés auxquels nous avons déjà abouti.

\section{Développement des corridors/secteurs de haute technologie}

Nos recherches ont permis de constater que le modèle de répartition des emplois hautement qualifiés doit être mis en relation avec d'autres critères. L'unité spatiale d'étude la plus appropriée est à ce sujet le niveau le plus petit statistiquement disponible, l'IRIS, à l'échelle communale. Les données susceptibles d'être exploitées à cette échelle sont cependant difficiles à obtenir et à traiter. En attendant une interprétation plus exhaustive, de notre part, de cette source de documentation, nous pouvons signaler l'indicateur des emplois hautement qualifiés, existant à l'INSEE dans le cadre des «fonctions métropolitaines supérieures» (Julien, 1996), dans les 11 branches suivantes (les \% indiquent, pour 1999, la part, en nombre d'emplois, de ces fonctions dans les branches respectives, soit $7,9 \%$ du total des emplois de l'ensemble de ces fonctions réunies en France, mais $10 \%$ a la CUS) : 


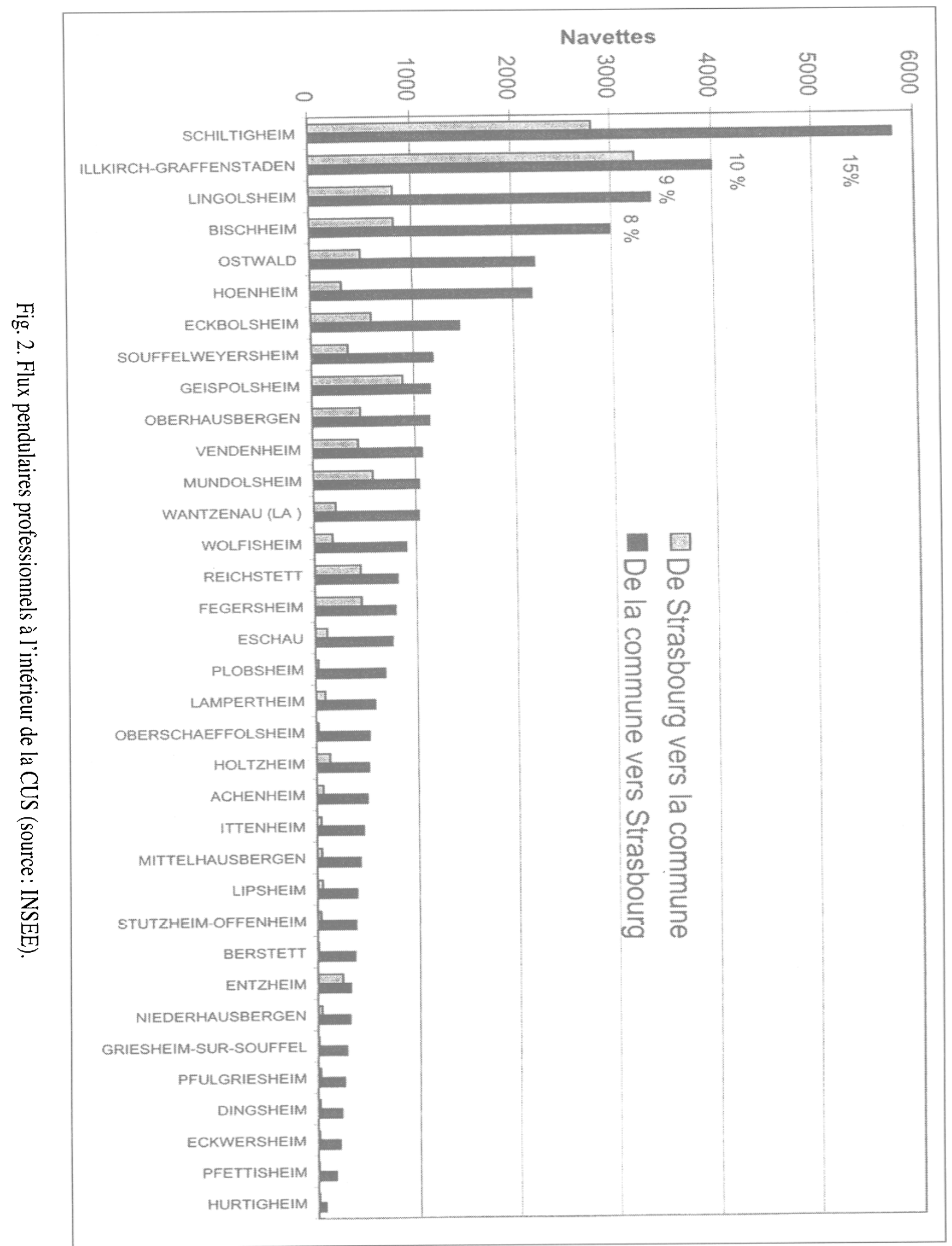




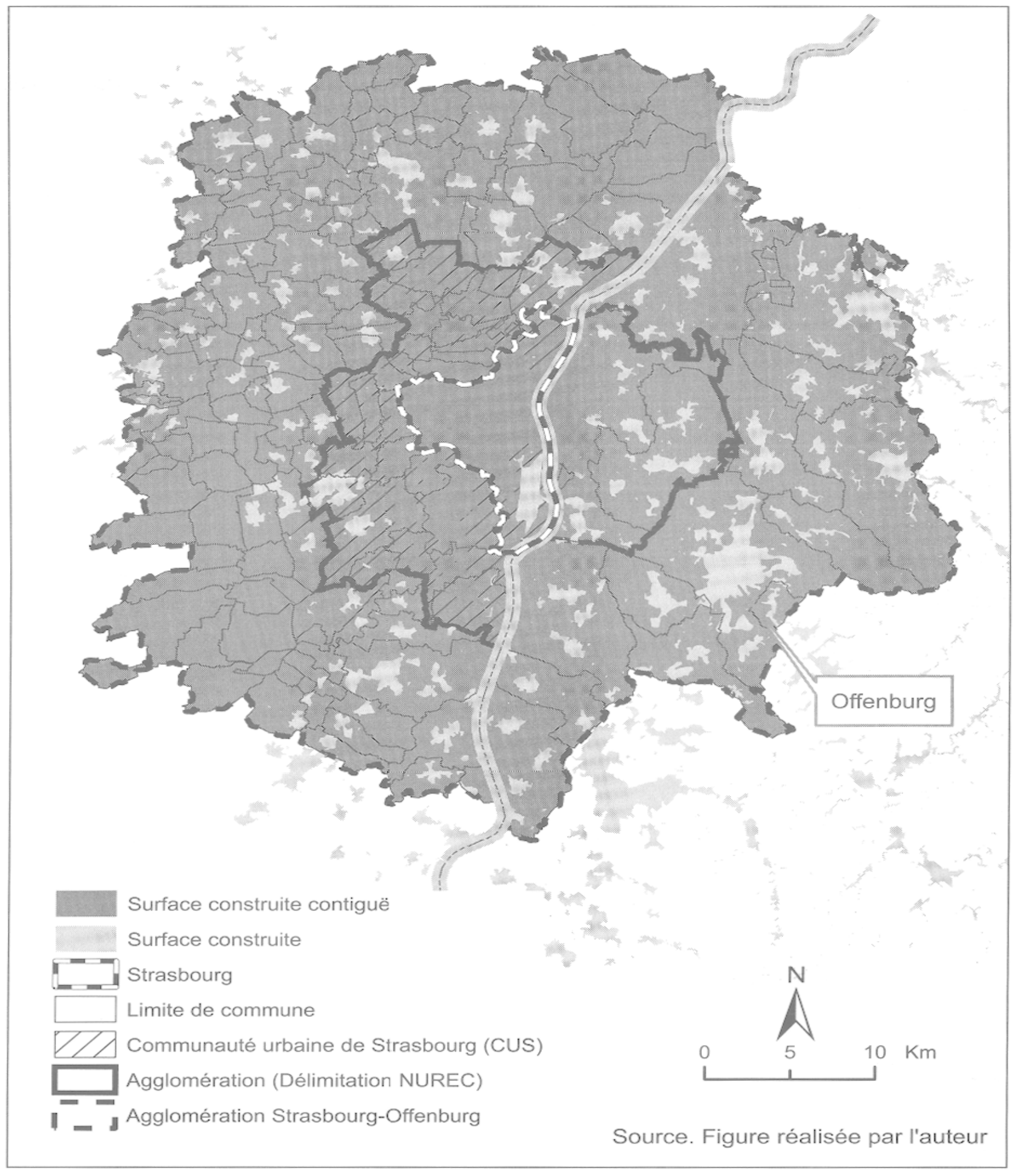

Fig. 3. Délimitation de l'agglomération de Strasbourg, selon le procédé NUREC. 


\begin{tabular}{|l|c|c|}
\hline Fonctions métropolitaines & $\begin{array}{c}\text { CUS } \\
\text { (total } \\
100 \%)\end{array}$ & $\begin{array}{c}\text { France } \\
\text { (total } \\
100 \%)\end{array}$ \\
\hline - art: & $5 \%$ & $7,2 \%$ \\
\hline - banque-assurance: & $11 \%$ & $8,1 \%$ \\
\hline - commerce: CUS/France & $12 \%$ & $11,9 \%$ \\
\hline - commercial dans l'industrie: & $3 \%$ & $4,5 \%$ \\
\hline - gestion: & $5 \%$ & $7,1 \%$ \\
\hline - information: & $2 \%$ & $3,2 \%$ \\
\hline - informatique: & $3 \%$ & $1,9 \%$ \\
\hline - recherche: & $23 \%$ & $15,2 \%$ \\
\hline - services aux entreprises: & $27 \%$ & $30,6 \%$ \\
\hline - télécommunications: & $4 \%$ & $4,2 \%$ \\
\hline - transports: & $6 \%$ & $5,9 \%$ \\
\hline
\end{tabular}

Avec plus de 23000 emplois répartis à l'intérieur de ces branches, Strasbourg occupe en 1999 la sixième place dans le contexte national. Cette ville dépasse même d'un quart d'emplois la moyenne des emplois des branches concernées des 50 aires urbaines françaises les plus importantes, à l'exception de Paris.

Tableau 3. Salariés actifs dans les «fonctions métropolitaines», selon le lieu de travail et le lieu de résidence.

\begin{tabular}{|l|c|c|c|c|}
\hline & \multicolumn{2}{|c|}{ Lieu de travail } & \multicolumn{2}{c|}{ Lieu de résidence } \\
\hline & 1990 & 1999 & 1990 & 1999 \\
\hline Strasbourg & 15037 & 15813 & 10073 & 11611 \\
\hline CUS & 19989 & 23238 & 16646 & 19605 \\
\hline
\end{tabular}

Source: INSEE.

L'offre dans le domaine des fonctions métropolitaines ne s'est toutefois pas élevée de manière significative à Strasbourg au cours de la décennie écoulée. Avec $17 \%$, la progression fut par contre significative dans la CUS hors Strasbourg (tableau 3 et figure 4).Cette augmentation est presque entièrement inhérente au déploiement du Parc d'innovation d'Illkirch-Graffensta- 
den. Le contraste est significatif entre les chiffres relatifs au lieu de travail et ceux propres au lieu de résidence. La part des salariés pendulaires dans les branches concernées par notre étude, domiciliés à l'extérieur de Strasbourg ou des autres communes de la CUS et migrant quotidiennement dans celle-ci, est particulièrement élevée: en 1999, elle a atteint environ $27 \%$ des migrants en direction de Strasbourg et environ $16 \%$ de ceux en direction des autres communes de la CUS.

\section{Aires de croissance et de concentration des fonctions métropoli- taines supérieures}

Remarque: compte tenu de l'échelle utilisée, la ville de Strasbourg n'a pas pu être représentée.

La ville de Strasbourg concentre toujours plus des deux tiers de tous les emplois dépendant des fonctions métropolitaines supérieures ( $75 \%$ en 1990, $68 \%$ en 1999). La diminution de ce taux en l'espace d'une décennie, de l'ordre de $10 \%$, s'est effectuée au bénéfice des autres communes de la CUS. La figure 4 montre toutefois que ce sont les communes à proximité immédiate

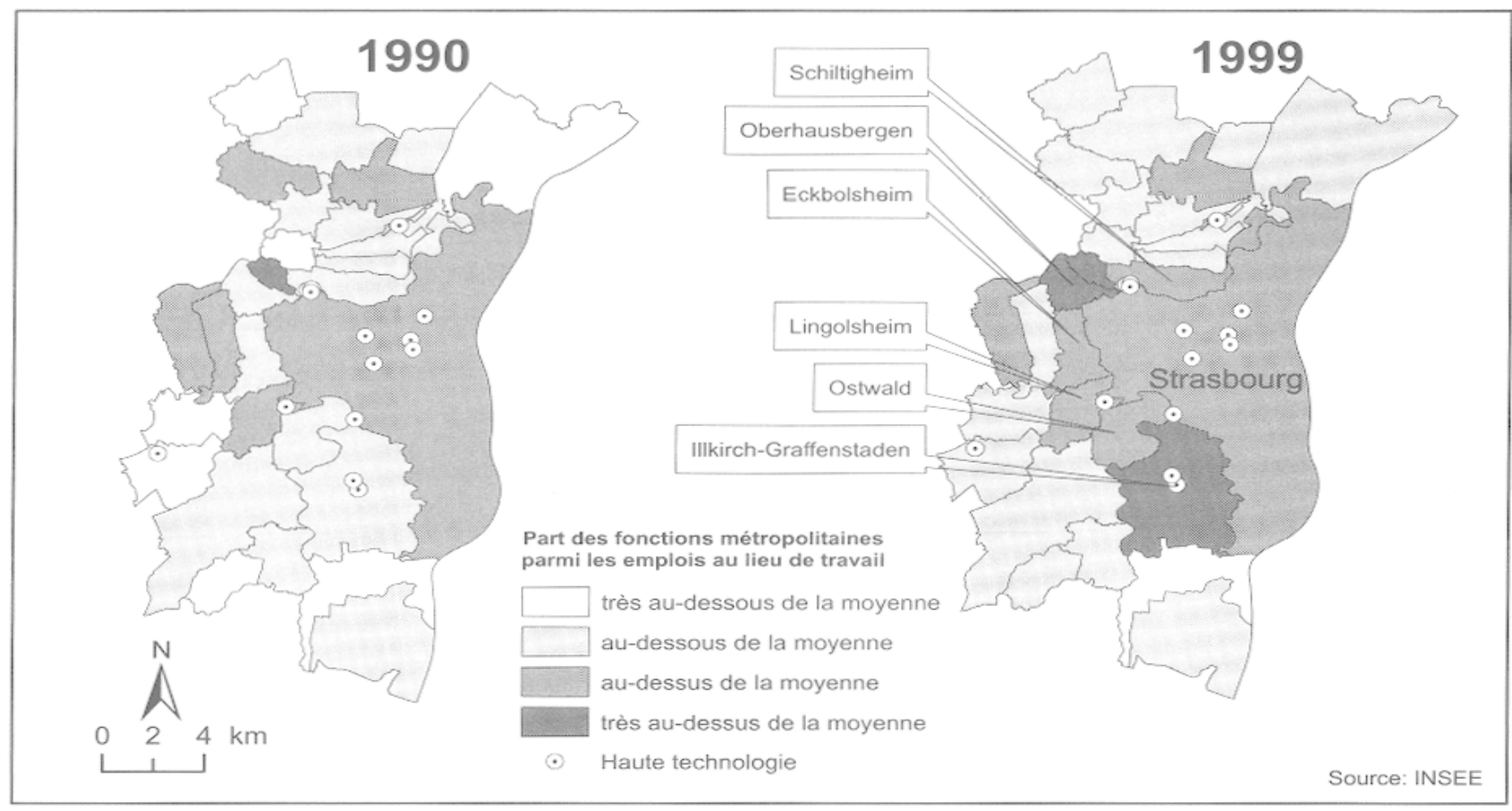

Fig. 4a. Part des fonctions métropolitaines parmi les emplois au lieu de travail. 


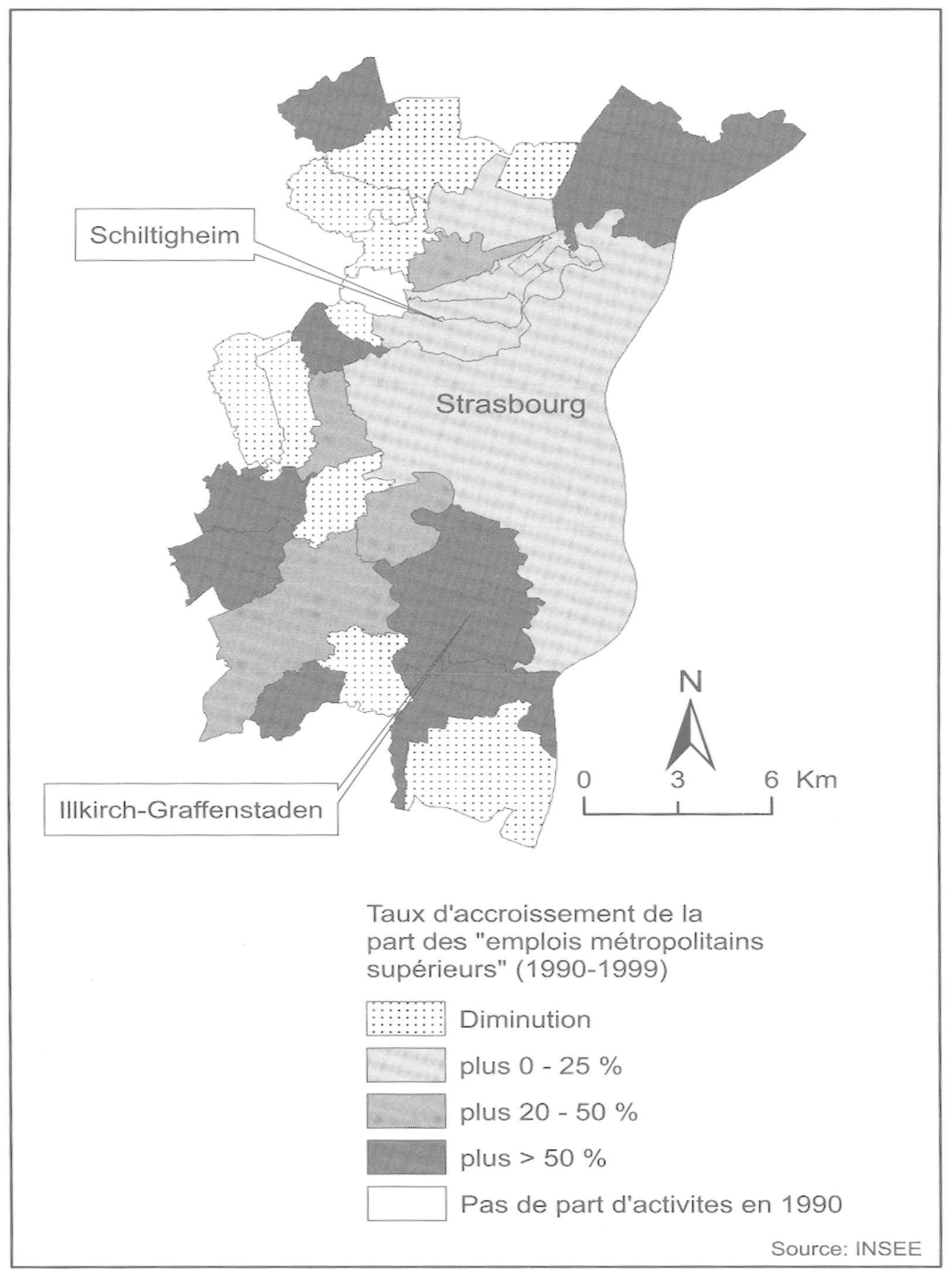

Fig. 4b. Taux d'accroissement de la part des «emplois métropolitains supérieurs». 


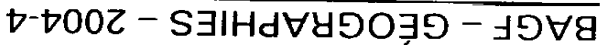

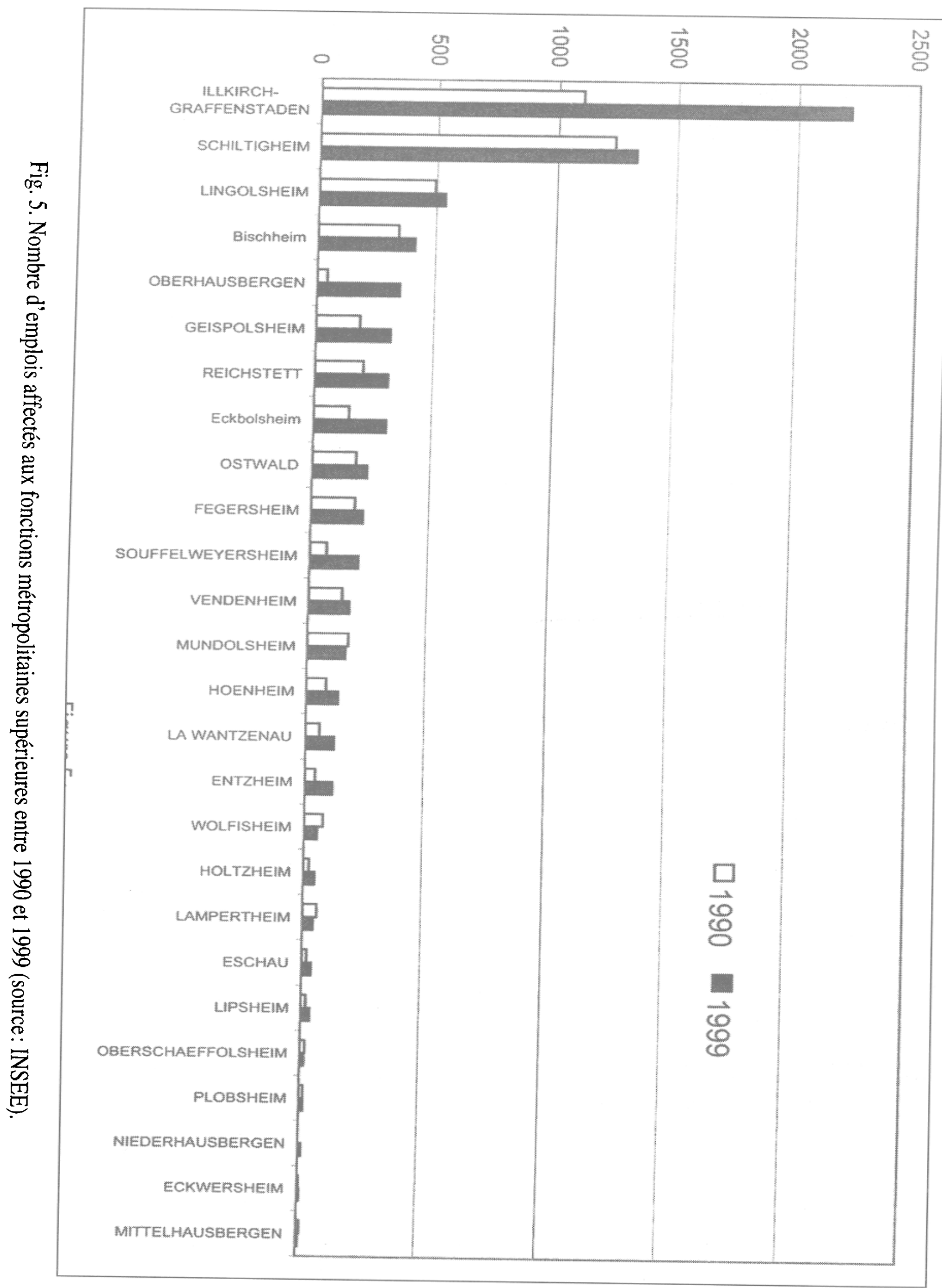


de Strasbourg qui détiennent les parts les plus élevées. Nous relevons à nouveau parmi elles les grandes communes d'Illkirch-Graffenstaden, de Schiltigheim et de Lingolsheim (tableau 4).

Tableau 4. Les trois communes jouxtant Strasbourg,

qui détiennent les taux d'emplois métropolitains supéricurs les plus élevés,

en comparaison avec cette ville (source: INSEE).

\begin{tabular}{|l|c|c|c|c|}
\hline & $\begin{array}{c}1990 \\
\text { au lieu de travail }\end{array}$ & $\begin{array}{c}1999 \\
\text { au lieu de travail }\end{array}$ & $\begin{array}{c}\text { Augmentation } \\
\text { en } \%\end{array}$ & $\begin{array}{c}\text { Part du total } \\
\text { des emplois } \\
\text { métropolitains } \\
\text { de la CUS }\end{array}$ \\
\hline Strasbourg & 15037 & 15813 & $+10 \%$ & $68 \%$ \\
\hline $\begin{array}{l}\text { Illkirch- } \\
\text { Graffenstaden }\end{array}$ & 1104 & 2218 & $+200 \%$ & $9,5 \%$ \\
\hline Schiltigheim & 1240 & 1330 & $+7 \%$ & $5,7 \%$ \\
\hline Lingolsheim & 492 & 535 & $+8 \%$ & $2,3 \%$ \\
\hline
\end{tabular}

Nous ne constatons guère une concentration claire de certaines fonctions métropolitaines sur des aires spécifiques de la CUS. La recherche $(23 \%)$ et les services aux entreprises ( $27 \%$ ) totalisent certes la moitié des emplois dans ce domaine, mais les autres branches sont néanmoins représentées aussi de façon marquante (figures 6 et 7). Nous assistons néanmoins à la concentration de certaines branches dans des communes ou sur des sites privilégiés, conformément au souhait des responsables publics.

Dans la mesure où il est possible de parler de la formation de corridors, nous pouvons considérer que c'est le cas à Schiltigheim et à Illkirch-Graffenstaden, supports possibles d'axes de développement. Même les projections d'aménagement de l'Ortenau visent une connexion au Parc d'innovation d'Illkirch. Il est ainsi tout à fait vraisemblable que se constituent des points d'ancrage dans le domaine de la recherche au le sud et au sud-ouest de la ville de Strasbourg.

En ce qui concerne la CUS, les emplois relatifs aux branches étudiées jouxtent essentiellement Strasbourg, participant à un processus de suburbanisation. Cette évolution est cependant contrée par le fait que les sites traditionnellement porteurs d'emplois hautement qualifiés sont localisés dans le centre élargi de Strasbourg (universités et fonctions européennes), une dynamique 


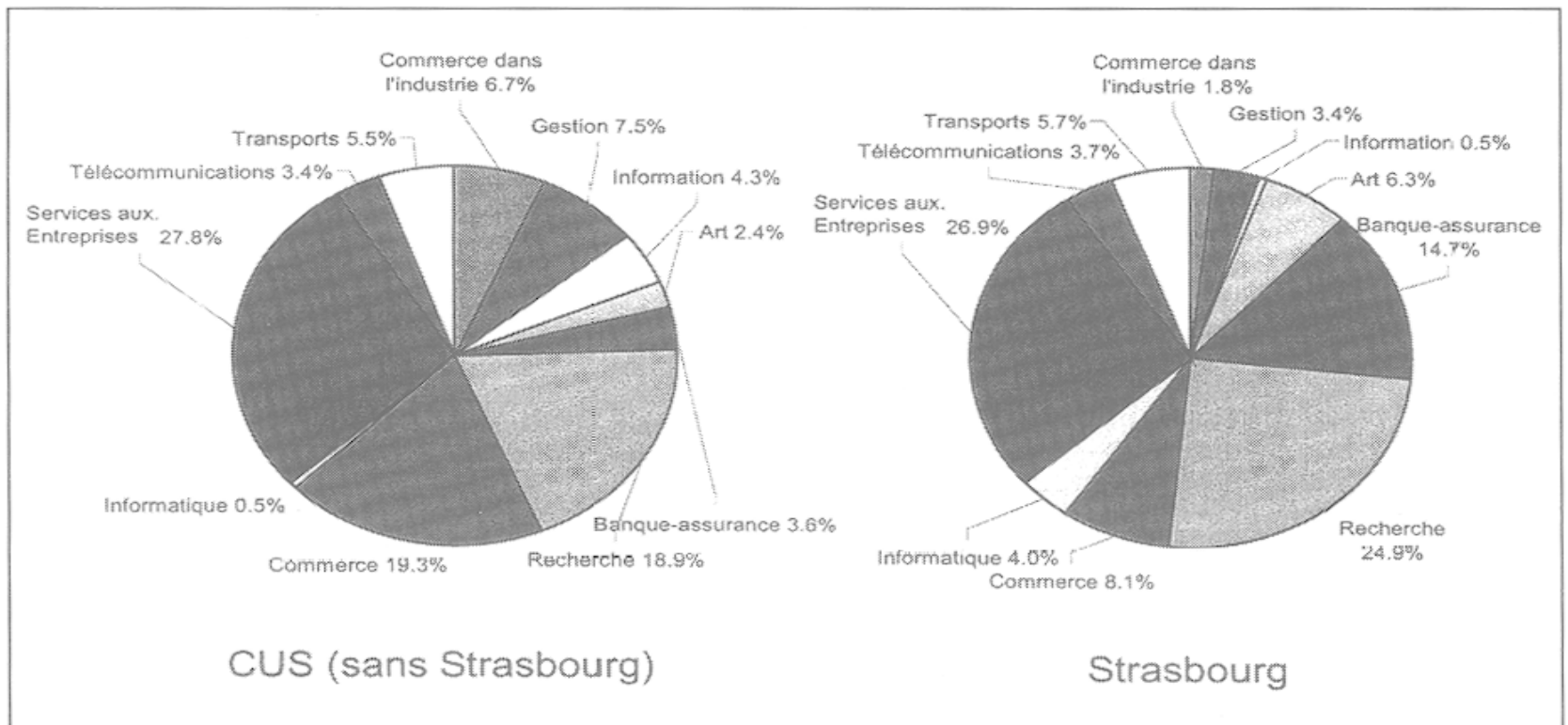

Fig. 6. Part des diverses fonctions métropolitaines supérieures dans la CUS (hors Strasbourg) et à Strasbourg en 1999

(source: INSEE).

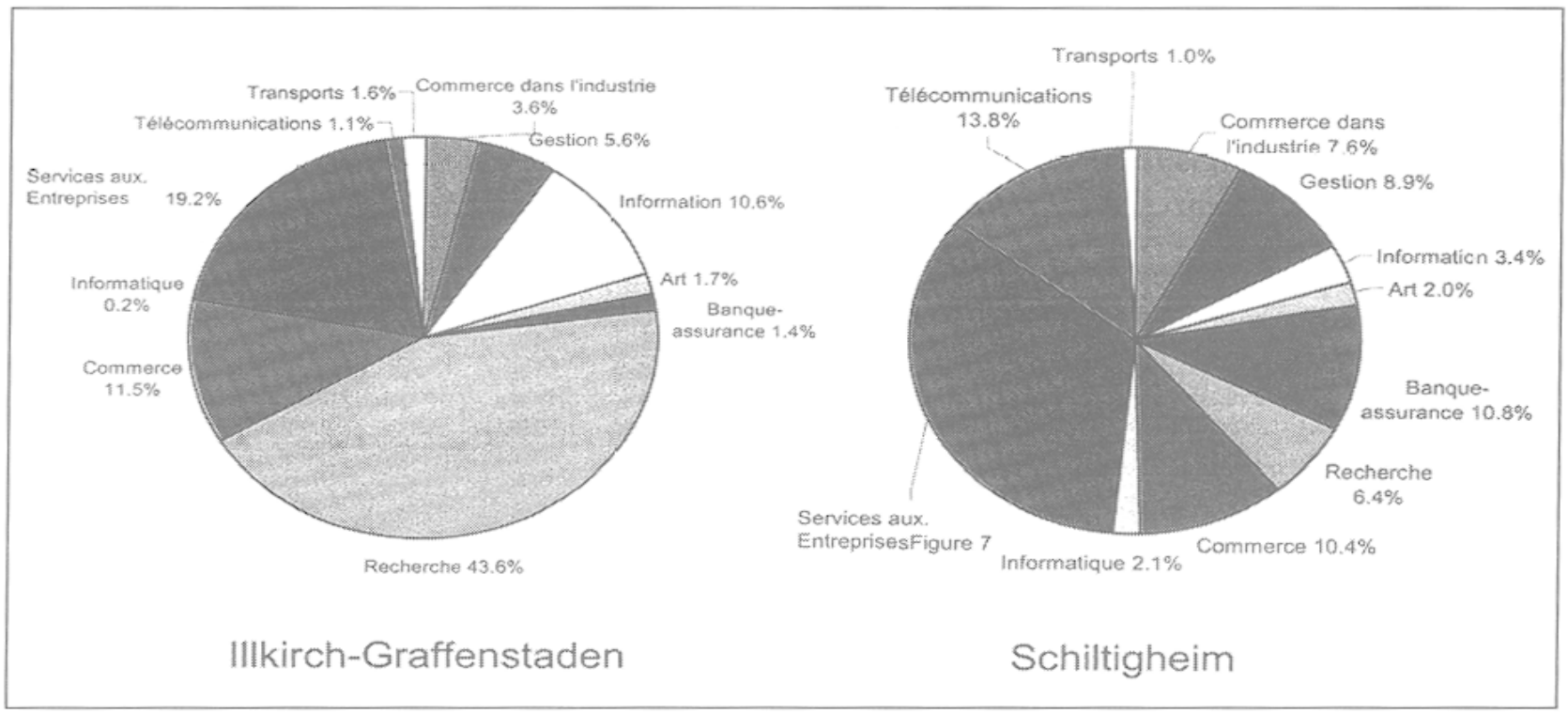

Fig. 7. La répartition en \% de certaines branches métropolitaines supérieures

à Illkirch-Graffenstaden et à Schiltigheim (1999). 
qui s'oppose au transfert de ces emplois dans les communes suburbaines. Si nous ne disposons pas encore, pour le moment, de données suffisamment précises sur la distribution de ces emplois à l'intérieur de la ville de Strasbourg, nous pouvons admettre dès à présent que ceux-ci sont localisés dans une large majorité en centre-ville élargi, ayant comme points d'appui essentiels la Place des Halles, les universités et le quartier de l'Europe.

En conclusion, nous constatons que le développement de l'agglomération de Strasbourg s'inscrit dans le cadre d'un aménagement régional, dans lequel l'Agence de développement et d'urbanisme de Strasbourg (ADEUS) joue à la fois un rôle de relais et de moteur, avec l'appui du pouvoir central parisien. C'est cette force d'entraînement qui, couplée au rôle de ville européenne, confère à Strasbourg et à son agglomération une dynamique qui rejaillit de façon de plus en plus prononcée sur la rive droite du Rhin, offrant à l'Ortenau des potentialités nouvelles de déploiement susceptibles de rejaillir à terme sur l'ensemble de l'agglomération binationale.

\section{Territoires urbains délaissés - Quartiers rejetés?}

\subsection{Aperçu des aires à problèmes en centre-ville}

Le développement présent des divers quartiers de la ville de Strasbourg s'effectue de façon très contrastée. Les quartiers à problèmes au point de vue socioéconomique et ceux dans lesquels les problèmes se multiplient ne forment pas de corridors, mais plutôt un «patchwork». Les quartiers considérés comme particulièrement problématiques sont ceux de Hautepierre et de Cronenbourg à l'ouest, mais aussi ceux de l'Elsau au sud et de la Cité de l'Ill au nord. Ces quartiers sont caractérisés par une diminution constante de la population et un certain vide résidentiel qui en résulte, au moment où le chômage augmente et où la problématique de la population étrangère rejoint davantage l'actualité.

Il est intéressant de constater que ces quartiers sont situés à une certaine proximité d'aires à hautes technologies, sans en être bénéficiaires; tel est par exemple le cas à Cronenbourg.

\subsection{Critères du manque d'intégration sociale et d'insertion aux circuits économiques}

Nous allons présenter sommairement quelques indicateurs essentiels permettant de mettre en relief l'évolution de la ville de Strasbourg vers une certaine dualité. Pour affiner notre analyse, nous comptons étudier par la suite les statistiques relatives à l'aide sociale, ainsi que celles concernant la criminalité. 
- La part des étrangers

A Strasbourg, de 1990 à 1999, le taux d'étrangers est passé de 10,3\% à 12,6\%. Dans la CUS par contre, il est tombé de 10,7 à 10,2\% (tableau 5).

Tableau 5. Evolution de la part des étrangers.

\begin{tabular}{|l|c|c|}
\hline Unité territoriale & 1990 & 1999 \\
\hline Strasbourg & $10,3 \%$ & $12,6 \%$ \\
\hline CUS & $10,7 \%$ & $10,2 \%$ \\
\hline Cronenbourg Ouest/Nord-ouest & $57,3 \%$ & $35,0 \%$ \\
\hline Polygone Sud & $40,6 \%$ & $39,4 \%$ \\
\hline Canardière Est-est & $39,4 \%$ & $28,4 \%$ \\
\hline Cronenbourg Ouest/Nord-est & $34,9 \%$ & $33,2 \%$ \\
\hline
\end{tabular}

Source: INSEE.

La pression étrangère a diminué simultanément dans les quartiers les plus marqués par elle. Les parts maximales d'étrangers continuent certes à être supérieures à la moyenne, avec environ 35 à $39 \%$, mais, par rapport à 1990, elles ont diminué sensiblement. En dépit de cette constatation, certains quartiers se transforment néanmoins en ghettos étrangers. La statistique officielle ne livre malheureusement pas de données sur l'origine de la population considérée comme française. En fait, un grand nombre d'immigrés nord-africains des seconde et troisième générations ont acquis la nationalité française au cours des dernières années. Quoiqu'ils n'apparaissent plus, bien sûr, comme faisant partie de la population étrangère, ils en ont souvent conservé le comportement et une certaine tendance à la ségrégation. Cette vague de naturalisation explique aussi en partie la diminution de la part de la population étrangère dans des quartiers comme Cronenbourg (fig. 8).

\section{- Le niveau de formation (fig. 9)}

La part des personnes ayant acquis un diplôme universitaire s'est accrue de 1990 à 1999. Ce groupe, ainsi que les ménages dont le chef est cadre ou engagé dans une activité de recherche-développement, a tendance à être très présent dans la zone suburbaine. Il est représenté dans une proportion au-dessus de la moyenne à la périphérie de la ville de Strasbourg, de même que dans un corridor très apparent s'étendant du centre-ville, par l'Orangerie, en direc- 


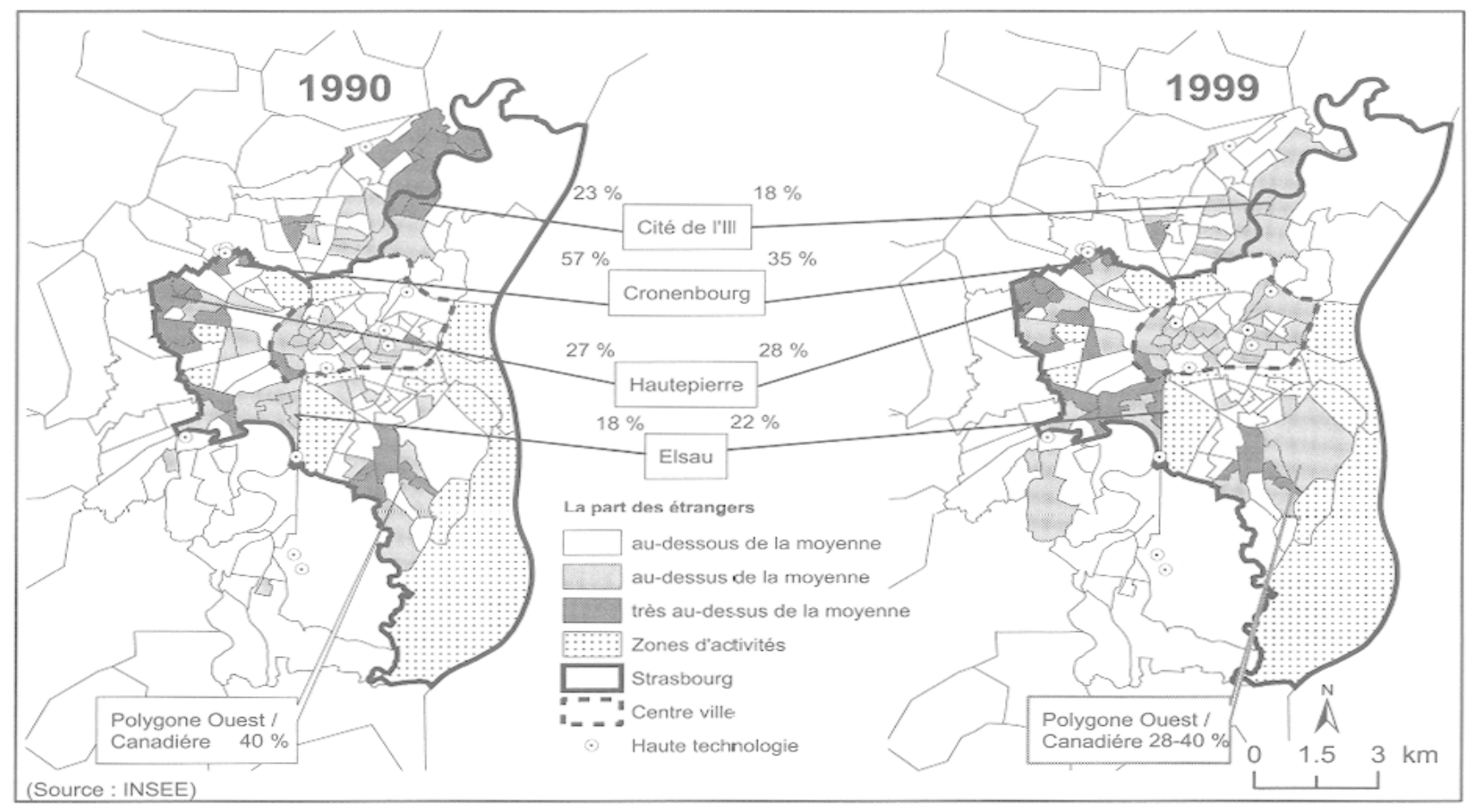

Fig. 8. La part des étrangers en 1990 et 1999 (source: INSEE).

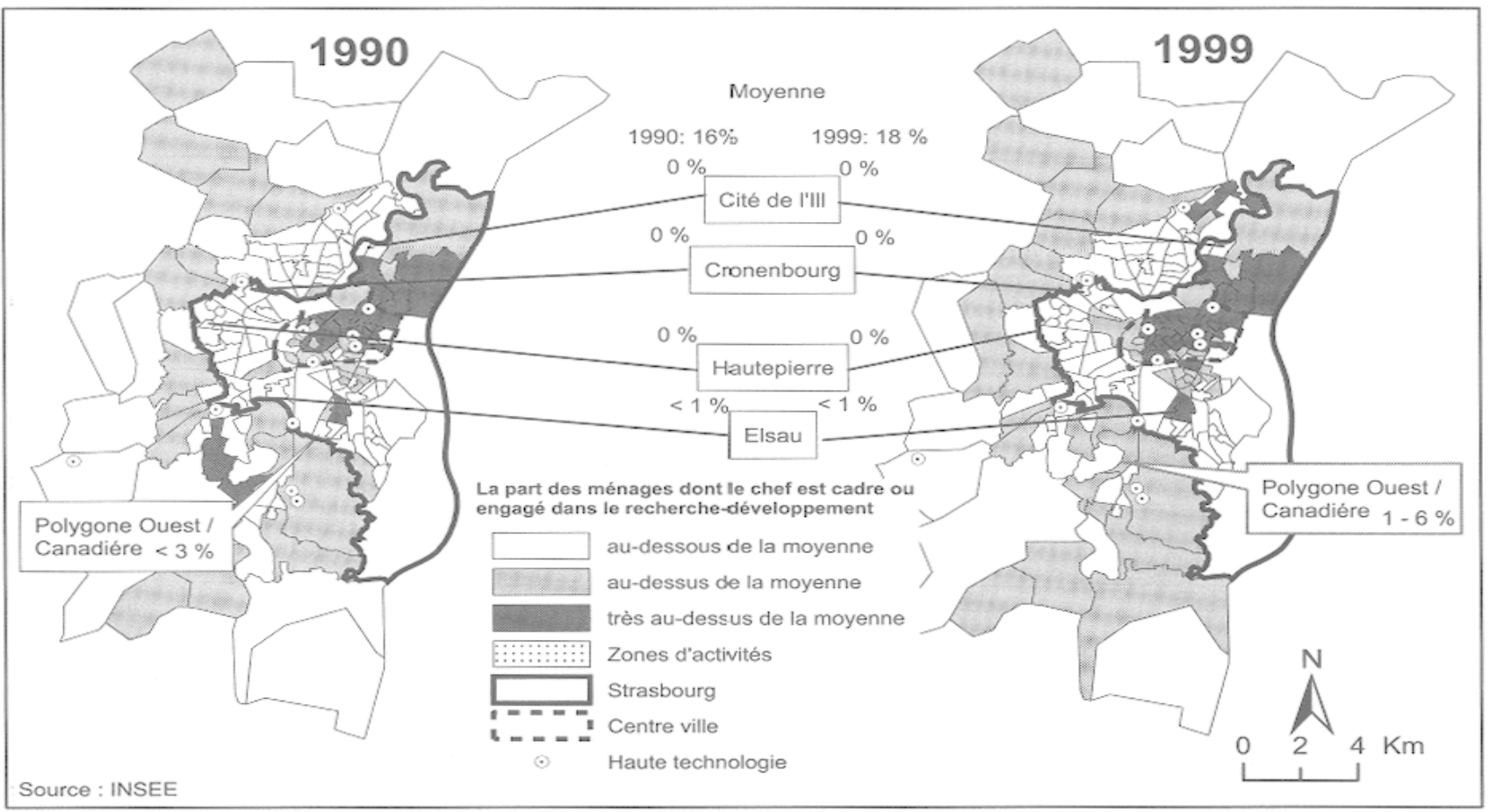

Fig. 9. La part des ménages dont le chef est cadre ou engagé dans la recherche-développement (source: INSEE). 
tion de la Robertsau, au nord-est. Dans ces quartiers ne résident, pour l'essentiel, que relativement peu d'étrangers. Dans les quartiers principalement peuplés par des étrangers, la part des diplômés universitaires est faible. Un peu partout le flou statistique résulte du fait que les naturalisés des seconde et troisième générations d'étrangers n'ont pas été répertoriés. Par ailleurs, les jeunes diplômés universitaires issus de l'immigration quittent très rapidement leur quartier d'origine.

\section{- Le chômage (fig. 10)}

Comme ailleurs, à Strasbourg, le chômagc s'cst accru de 1990 à 1999: de $10,8 \%$ en 1990 , il a atteint $13,0 \%$ en 1999 . Cette évolution est caractérisée par une concentration spatiale dans les quartiers «chauds» de Hautepierre, Cronenbourg, à l'Elsau et dans la Cité de l'Ill, des quartiers où est également concentrée la population étrangère. Les quartiers résidentiels occupés par la population à niveau de formation plus élevé par contre échappent à cette empreinte du chômage.

- Les femmes salariées seules, chargées d'enfant(s) (fig. 11)

La part de ces femmes n'a progressé que de $1,8 \%$ de 1990 à 1999, au regard de la population totale. Nous observons cependant que ces femmes ont tendance à se concentrer sur des aires résidentielles défavorables, au mieux moyennes.

\section{Perspectives différenciées des quartiers}

Les quartiers à graves problèmes sociaux - Hautepierre, grands ensembles de Cronenbourg et de l'Elsau - demeurent à l'écart de l'évolution générale de Strasbourg. Ce contraste est également perceptible à Schiltigheim, ville de la CUS jouxtant Strasbourg au nord. La plupart des autres communes de la CUS sont toutefois largement intégrées à l'évolution générale, positive.

Les distorsions sont d'autant plus choquantes, tant à Cronenbourg qu'à Schiltigheim, que les aires défavorisées sont au voisinage d'aires sur lesquelles se concentrent d'importantes unités de recherche de pointe. L'insuffisance statistique ne permet pas de savoir actuellement dans quelle mesure le centre-ville est affecté par de tels contrastes à l'échelle micro-spatiale. 


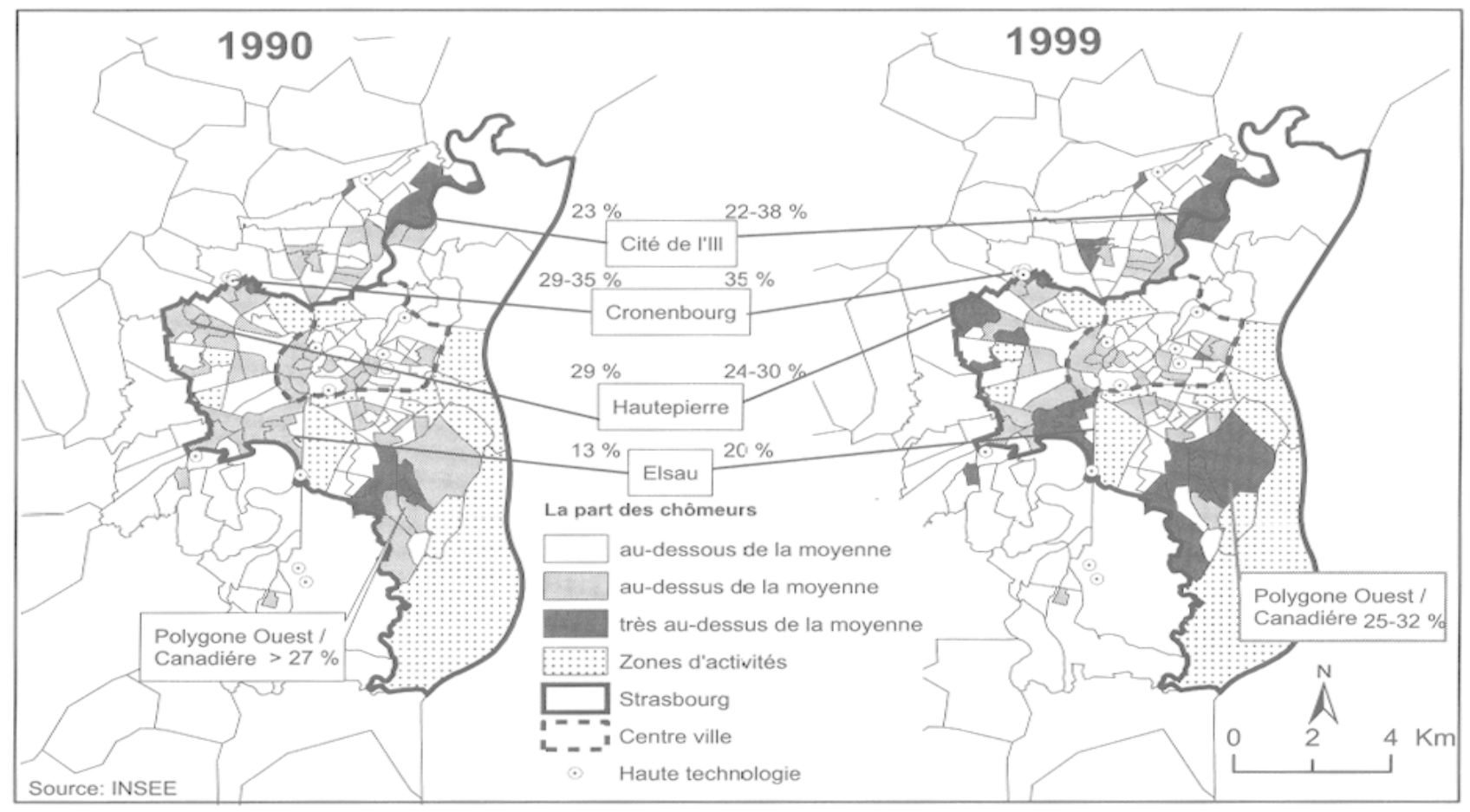

Fig. 10. Le chômage.

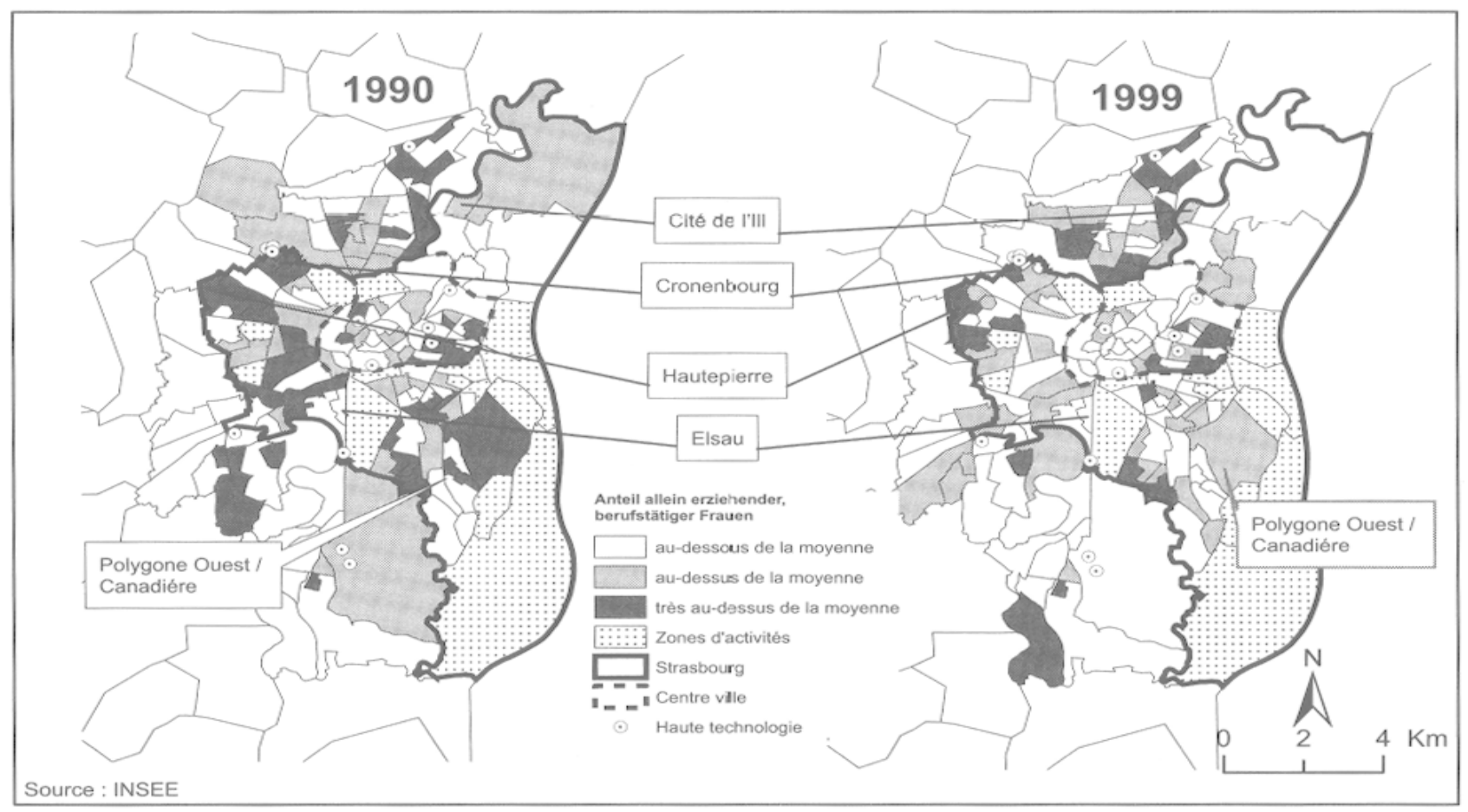

Fig. 11. Part des femmes salariées seules, chargées d'enfant(s). 


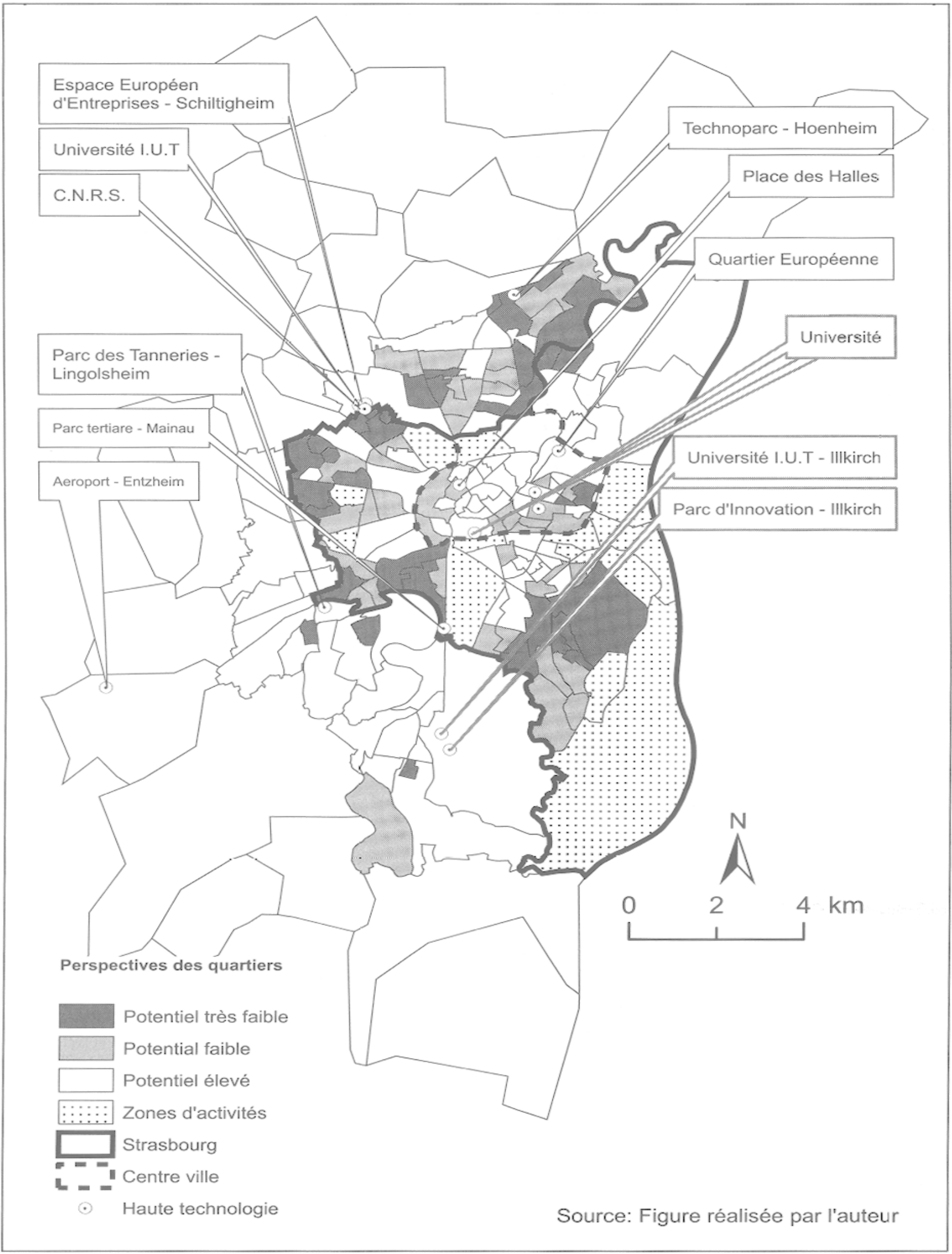

Fig. 12. Perspectives différenciées des quartiers. 
La situation présente soulève les questions suivantes:

- Dans quelle mesure les équipements de hautes technologies exercent-ils une influence positive sur leur environnement immédiat?

- Les hautes technologies sont-elles vraiment un moyen efficace susceptible de conforter de manière durable la totalité de l'espace socioéconomique?

- L'implantation volontariste d'unités de haute technologie entraînant la création d'emplois de pointe conduit-elle à l'évolution vers une ville duale, une ville sans classe moyenne?

Il ne saurail être question de mettre en cause les résultats socio-économiques globalement très positifs des mesures prises à Strasbourg en matière d'aménagement du territoire. Il importe néanmoins de s'interroger sur les effets éventuellement pervers de l'évolution en cours. Les indices de fragmentation et de polarisation d'une certaine ségrégation relevés au cours de notre investigation méritent une attention suivie portée au phénomène du développement dual: tandis que l'agglomération de Strasbourg bénéficie d'un développement croissant qui la conduit vers des structures modernes, la transformation en cours ne permet pas à l'ensemble des secteurs spatiaux de bénéficier des retombées en matière de qualification professionnelle, de niveau et de rythme de vie; une réelle marginalisation socio-spatiale est esquissée, dont les remèdes se font attendre.

\section{RÉFÉRENCES BIBLIOGRAPHIQUES}

AYDALOT P., 1986. - Milieux innovateurs en Europe, Paris, Gremi, 386 S.

BEHREND H., KRUSE C., 2001. - «Die Europäische Metropolregion Zürich - die Ent-stehung des subpolitischen Raumes», in : Geographica Helvetica, 3(56)2001, S. 202-213.

BLOTEVOGEL H.H., 2001. - «Die Metropolregionen in der Bundesrepublik Deutschland ein neues strategisches Raumbild?», in: Geographica Helvetica, 3(56)2001, S. 157-169.

BUTZIN B., 2000a. - «Netzwerkansätze in der Regionalentwicklung», in: Zeitschrift für Wirtschaftsgeographie, 3/4(44)2000, S. 145-148.

BUTZIN B., 2000b. - «Netzwerke, kreative Milieus und lernende Region. Perspektiven für die regionale Entwicklungsplanung?», in: Zeitschrift für Wirtschaftsgeographie, 3/4(44)2000, S. $149-166$.

FLOETING H., 1996. - «Teleports - High-Tech-Oasen in der Stadtentwicklungswüste?», in: Wechselwirkung - Technik, Naturwissenschaft, Gesellschaft, Nr. 66, S. 13-16.

GRASSL H., 2000. - Strukturwandel der Arbeitsteilung. Globalisierung, Tertiarisierung und Feminisierung der Wohlfahrtsproduktion, Konstanz, Universitätsverlag Konstanz.

JULIEN P., 1996. - «Spécificités des grandes villes de province», Economie et statistique, $\mathrm{n}^{\circ} 294-295,1996,4 / 5, \mathrm{~S} .137-152$.

JULIEN P., 2002. - «Onze fonctions pour qualifier les grandes villes», INSEE Première, $n^{\circ} 840$, Mars 2002. 
KEIM R.,1999. - «Wohnungsmarkt und soziale Ungleichheit - Über die Entwicklung städtischer Polarisierungsprozesse», Stadtforschung aktuell, Bd. 72, Basel, Berlin, Boston, Birkhäuser Verlag, $340 \mathrm{~S}$.

KNUTH M., SCHRÄPLER J.-P., SCHUMANN D., 2001. - Die Neuverteilung von Beschäfti gungschancen und - risiken in der Dienstleistungsgesellschaft, Institut für Arbeit und Technik, Graue Reihe 2001-01.

KOMMISSION FÜR ZUKUNFTSFRAGEN DER FREISTAATEN BAYERN UND SACHSEN, 1996. - Erwerbs tätigkeit und Arbeitslosigkeit in Deutschland. Entwicklung, Ursachen und Maflnah men, Teil I: Entwicklung von Erwerbstätigkeit und Arbeitslosigkeit in Deutschland und anderen frühindustrialisierten Ländern, Bonn.

KOSCHATZKY, K., KULICKE, M., ZENKER A. (ed.), 2001. - Innovation Networks. Concepts and challenges in the European perspective, Heidelberg, Physica-Verlag.

KRÄTKE S., 1991. - «Cities in transformation - The case of West-Germany », in: Benko \& Dunfords (eds.), Industrial Change and regional Development - the transformation of new industrial spaces, London, New York, Belhaven Press, S. 250-272.

KULKE E., 1998. - Wirtschaftsgeographie Deutschlands, Stuttgart, Klett-Perthes.

MEIER, J., BECK R., 2000. - Allgemeine Industriegeographie, Stuttgart, Klett-Perthes.

NONN H., 1999. - Villes et aménagement régional en Alsace, La Documentation Française, Paris.

O'LOUGHLIN J., FRIEDRICHS J., 1996. - «Polarization in post-Industrial societies - Social and economic roots and consequences», in: O'LOUGHLIN J. \& FRIEDRICHS J., 1996 (eds.), Polarization in post-Industrial metropolises, Berlin, New York, de Gruyter, S. 1-18.

RIFKIN J., 1995. - Das Ende der Arbeit und ihre Zukunft, Frankfurt a. Main, New York, Campus.

SCHERF K., 1998. - Die metropolitane Region Berlin - Genese und Niedergang, Revitalisierung und Innovation, IRS-Forschungsberichte, Heft 17.

SCHMITZ S., 2001. - Revolution der Erreichbarkeit - Gesellschaft, Raum und Verkehr im Wandel. - Stadtforschung aktuell, Bd. 83, Opladen, Leske + Budrich, $320 \mathrm{~S}$.

STRAMBACH S., 2002. - "Change in the innovation process. New knowledge production and competitive cities - the case of Stuttgart", European Planning Studies, Vol. 10, No.2.

WACKERMANN G., 2000. - «Das Elsass - Wandel und Perspektiven einer europäischen Grenzregion», in: Geographica Helvetica, 1(55)2000, S. 45-60.

WACKERMANN G., 2001. - «Urbaner Wandel und soziale Fragmentierung - Einleitung zum Themenheft», in: Geographica Helvetica, 4(56)2001, S. 230-233. 